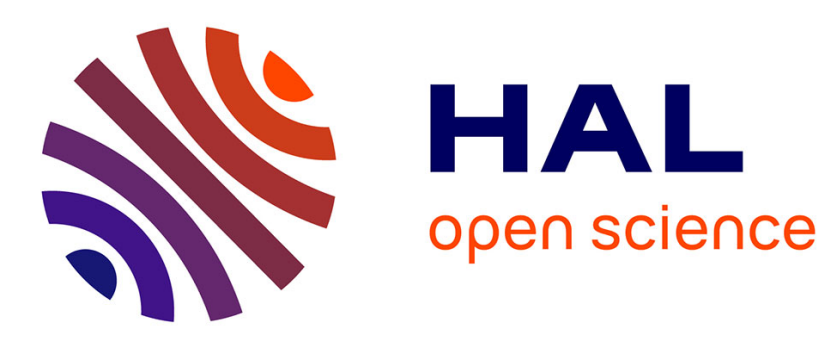

\title{
Numerical simulations of a transport-aircraft configuration
}

Eric Goncalvès da Silva, Robert Houdeville

\section{To cite this version:}

Eric Goncalvès da Silva, Robert Houdeville. Numerical simulations of a transport-aircraft configuration. International Journal of Computational Fluid Dynamics, 2009, 23 (6), pp.449-459. 10.1080/10618560903107452 . hal-00530291

\section{HAL Id: hal-00530291 \\ https://hal.science/hal-00530291}

Submitted on 26 Feb 2017

HAL is a multi-disciplinary open access archive for the deposit and dissemination of scientific research documents, whether they are published or not. The documents may come from teaching and research institutions in France or abroad, or from public or private research centers.
L'archive ouverte pluridisciplinaire HAL, est destinée au dépôt et à la diffusion de documents scientifiques de niveau recherche, publiés ou non, émanant des établissements d'enseignement et de recherche français ou étrangers, des laboratoires publics ou privés. 


\title{
RESEARCH ARTICLE
}

\section{Numerical simulations of a transport-aircraft configuration}

\author{
E. Goncalves ${ }^{a *}$ and R. Houdeville ${ }^{b}$ \\ ${ }^{a}$ LEGI, 1025 rue de la Piscine, 38400 St Martin d'Heres, France; ${ }^{b}$ ONERA-Toulouse, 2 avenue \\ Edouard Belin, 31000 Toulouse, France
}

(Received 00 Month 200x; final version received 00 Month 200x)

\begin{abstract}
An implicit low-cost Navier-Stokes solver combined with a multigrid algorithm and wall functions has been developed for efficient numerical simulations on a realistic wing-body aircraft configuration. A study of the behavior of different transport-equation turbulence models is given. Comparisons are made with experimental data. The structure of the three-dimensional flow separation predicted by computations is described and its topological coherence is checked.
\end{abstract}

Keywords: CFD; aerodynamics; turbulence models; 3D separation; vortical structures

\section{INTRODUCTION}

During the last decade, considerable progress has been made in the development and validation of numerical simulation solvers for complex aerodynamic applications. Today, advanced numerical tools are intensively used in the design process of aerospace components which involve three-dimensional turbulent flows with separation. However, CFD codes still suffer from deficiencies in representability of computations with respect to the physics, from the lack of accuracy and robustness and from large CPU costs. Indeed, despite the computers growing capacity, the resolution of the Reynolds-Averaged Navier-Stokes (RANS) equations coupled with a transport-equation turbulence model, integrated down to the wall, for a complete aircraft configuration remains expensive.

In order to reduce the CPU cost, different strategies have been explored : implicit methods, multigrid algorithm and parallel computing. Another possibility to avoid the full Navier-Stokes resolution is the use of wall functions as boundary conditions. Thanks to the robustness improvement, the quality of results in two-dimensional separated flows and the CPU cost saving, the wall law approach is a promising method (Mohammadi and Pironneau 1997, Craft et al. 2004, Kalitzin et al. 2005). However, the existence of a law of the wall for three-dimensional flows is still an open issue. Olcmen (Olcmen and Simpson 1992) has investigated the possible existence of a universal velocity profile in three-dimensional boundary layers and has concluded that there is not strong evidence for such a 3D turbulent boundary layer velocity profile, neither for the streamwise component nor for the transversal component. In the numerical study of the flow around an ellipsoid, Tsai and Withney (Tsai and Whitney 1999) used a logarithmic law for the streamwise direction but changed the value of the constant to obtain a good agreement

${ }^{*}$ Corresponding author. Email: eric.goncalves@legi.grenoble-inp.fr

ISSN: $1029-8436$ print/ISSN $1477-268 \mathrm{X}$ online

(C) 2008 Taylor \& Francis

DOI: 10.1080/1029843YYxxxxxxxx

http://www.informaworld.com 
with the experimental data.

In the present study, we propose a low-cost implicit solver coupled with a multigrid algorithm and the use of a wall law approach to investigate the 3D flow around a wing-body configuration. Moreover, the behavior of four popular turbulence models is compared. This configuration has been a European test-case to validate CFD codes and turbulence modeling. It was computed by LeBalleur (LeBalleur et al. 1997) using a defect-formulation theory and a 3D thin-layer approach. RANS computations have been performed with the Baldwin-Lomax model (Elsholtz and John 1997), the Granville algebraic model, the one-equation Wolsthein model and the Chen-Patel two-layer model (Tourrette 1996) and also with an improved $k-\omega$ model (Kroll et al. 2000). A study of a drag polar has been realized with the $k-\omega$ linearized algebraic stress (LEA) model (Rakowitz et al. 2003). Recently computations with explicit algebraic Reynoldsstress models (EARSM) have been performed and attention was drawn to the leading-edge separation pocket on the outboard part of the wing (Franke et al. 2005). Regarding the shock location, algebraic models can not give predictions with an acceptable level of accuracy. At least a transport-equation model, which takes into consideration history effects, is required to accurately model 3D flow phenomena.

Finally, a study of the wing-fuselage junction flow is proposed. The adverse pressure gradient in the streamwise direction imposed by the wing often causes the upwind boundary layer on the body to separate and to form a horseshoe vortex around the wing. On the trailing edge, the horseshoe vortex feeds a vortical structure, called tornado vortex. The topological coherence of numerical vortices is checked based on the critical-point theory.

\section{GOVERNING EQUATIONS AND TURBULENCE MODELS}

\section{$2.1 \quad$ Reynolds-Averaged Navier-Stokes equations}

The compressible RANS equations coupled with a two-equation turbulence model can be expressed as :

$$
\begin{gathered}
\frac{\partial w}{\partial t}+\operatorname{div}\left(F_{c}-F_{v}\right)=S \\
w=\left(\begin{array}{c}
\rho \\
\rho V \\
\rho E \\
\rho k \\
\rho \Psi
\end{array}\right) \quad ; \quad F_{c}=\left(\begin{array}{c}
\rho V \\
\rho V \otimes V+p \overline{\bar{I}} \\
(\rho E+p) V \\
\rho k V \\
\rho \Psi V
\end{array}\right) \quad ; \quad F_{v}=\left(\begin{array}{c}
0 \\
\overline{\overline{\tau^{v}}}+\overline{\overline{\tau^{t}}} \\
\left.\overline{\overline{\tau^{v}}}+\overline{\overline{\tau^{t}}}\right) \cdot V-q^{v}-q^{t} \\
\left(\mu+\mu_{t} / \sigma_{k}\right) \operatorname{grad} k \\
\left(\mu+\mu_{t} / \sigma_{\Psi}\right) \operatorname{grad} \Psi
\end{array}\right)
\end{gathered}
$$

where $w$ denotes the conservative variables, $F_{c}$ and $F_{v}$ the convective and viscous flux densities and $S$ the source terms which concern only the transport equations. $\Psi$ is the length scale determining variable.

The exact expression of the eddy viscosity $\mu_{t}$ and the source terms depends on the turbulence model, as well as the constants $\sigma_{k}$ and $\sigma_{\Psi}$.

The total stress tensor $\overline{\bar{\tau}}$ is evaluated following the Stokes hypothesis and the Boussinesq assumption. The total heat flux vector $q$ is obtained from the Fourier law with the constant Prandtl 
number hypothesis.

$$
\begin{aligned}
& \overline{\bar{\tau}}=\overline{\overline{\tau^{v}}}+\overline{\overline{\tau^{t}}}=\left(\mu+\mu_{t}\right)\left[\frac{1}{2}\left(\operatorname{grad} V+(\operatorname{grad} V)^{t}\right)-\frac{2}{3}(\operatorname{div} V) \overline{\bar{I}}\right]+\frac{2}{3} k \overline{\bar{I}} \\
& q=q^{v}+q^{t}=-\left(\frac{\mu}{P_{r}}+\frac{\mu_{t}}{P_{r t}}\right) C_{p} \operatorname{grad} T
\end{aligned}
$$

The viscosity is determined by the Sutherland law.

\section{$2.2 \quad$ Turbulence Models and Wall law approach}

Various two-equation turbulence models are used in the present study : the Smith $k-l$ model (Smith 1994), the Wilcox $k-\omega$ model (Wilcox 1988), the Menter SST $k-\omega$ model (Menter 1993, 1994) and also the one-equation Spalart-Allmaras (Spalart and Allmaras 1992).

At the wall, a no-slip condition is used coupled to a wall law treatment. It consists in imposing the diffusive flux densities, required for the integration process, in adjacent cells to a wall. The shear stress $\tau$ and the heat flux $q$ are obtained from an analytical velocity profile

$$
\begin{aligned}
& \bar{u}^{+}=y^{+} \quad \text { if } y^{+}<11.13 \\
& \bar{u}^{+}=\frac{1}{\kappa} \ln y^{+}+5.25 \text { if } \quad y^{+}>11.13 \\
& \bar{u}^{+}=\bar{u} / U_{\tau} \quad ; \quad y^{+}=\frac{y U_{\tau}}{\nu_{w}}
\end{aligned}
$$

and from the integration of the total enthalpy equation, in which the convection is neglected

$$
u \tau_{x y}-q_{y}=-q_{w}
$$

where $x$ and $y$ denote here the longitudinal and normal direction with respect to the wall.

In equation $(4), \bar{u}$ represents the van Driest $(1951,1957)$ transformed velocity for compressible flows

$$
\bar{u}=\int_{0}^{u} \sqrt{\frac{\rho}{\rho_{w}}} \mathrm{~d} u
$$

For an adiabatic wall, integration of equations (5) and (6) gives

$$
\begin{aligned}
T_{w}-T & =A \frac{u^{2}}{2} \quad ; \quad A=\frac{\mu+\mu_{t}}{C_{p}\left(\frac{\mu}{P_{r}}+\frac{\mu_{t}}{P_{r_{t}}}\right)} \\
\bar{u} & =\frac{1}{\sqrt{B}} \arcsin (\sqrt{B} u) \quad ; \quad B=\frac{A}{2 T_{w}}
\end{aligned}
$$

The wall law treatment is now straightforward. Knowing $u$ from the Navier-Stokes solution, relationships (7) and (8) give $\bar{u}$. The shear stress value is obtained from (4) and is assumed constant in the wall normal direction. To compute boundary layer separation, the wall law is expressed in a reference frame defined by the velocity direction in adjacent cells to a wall. Such a treatment is not in contradiction with the fact that the log law does not exist in separated regions. Actually, in these regions, $\tau_{w}$ remains small and therefore $y^{+}$is small, leading to the use of the linear part of the velocity profile. This is equivalent to computing the velocity gradient 
over two points instead of three for the ordinary cells.

For three-dimensional boundary layers, the existence of a wall law is assumed valid for the streamwise velocity component. Moreover, in adjacent cells to a wall, the velocity is supposed colinear to the wall friction direction. This is the only assumption made regarding the transversal velocity component.

As concerns the transport equations of the turbulence models, $k$ is set to 0 at the wall and its production is imposed according to the formulation proposed by Viegas and Rubesin (Viegas and Rubesin 1983, 1985) where the thickness of the viscous sub-layer $y_{v}^{+}$is assumed constant

$$
\left(P_{k}\right)_{1}=\frac{\tau_{w}^{3 / 2}}{2 \kappa y_{1} \sqrt{\rho_{w}}} \ln \frac{2 y_{1}}{y_{v}}
$$

For the two-equation models, the second variable is deduced from an analytical relation and is imposed in adjacent cells to a wall. The characteristic length scale of the Chen model (Chen and Patel 1988) is used for the specific dissipation $\omega$ and a classical linear law for the length $l$.

For the one-equation Spalart-Allmaras model, the transported quantity is imposed in adjacent cells to a wall by using the closure relations of the model, the velocity profile and a mixing-length formulation for the eddy viscosity.

More details concerning the wall law approach are given in reference (Goncalves and Houdeville 2001).

\section{NUMERICAL METHODS}

The numerical simulations were carried out using an implicit CFD code solving the uncoupled RANS/turbulent systems for multi-domain structured meshes. This solver is based on a cellcentered finite-volume discretization.

\subsection{Spatial discretization}

The system in integral form is written for a computational cell of volume $\Omega$ limited by a surface $\Sigma$ and with an outer normal $n$. These equations can be expressed as :

$$
\frac{d}{d t} \int_{\Omega} w d \Omega+\oint_{\Sigma} F_{c} \cdot n d \Sigma-\oint_{\Sigma} F_{v} \cdot n d \Sigma=\int_{\Omega} S d \Omega
$$

Using the finite-volume technique for space discretization, a semi-discrete form of equations may be written as:

$$
\Omega \frac{\partial w}{\partial t}+\sum_{\text {allfaces }}\left(F_{c}-F_{v}\right) \cdot n \Sigma=\Omega S
$$

For the mean flow, the convective flux density vector on a cell face is computed with the space-centered Jameson scheme stabilized by a scalar artificial dissipation (Jameson et al. 1981). The dissipative operator comprises second (parameter $k_{2}$ ) and fourth (parameter $k_{4}$ ) order differences.

Discretization of the viscous terms is performed by a second-order space-centered scheme. 
For the turbulence transport equations, the upwind Roe scheme (Roe 1981) was used to obtain a more robust method. The second-order accuracy was obtained by introducing a flux-limited dissipation (Tatsumi et al. 1995).

\subsection{Temporal discretization}

Time integration is achieved through a low-cost implicit method (Luo et al. 1998, 2001). The implicit method consists in solving a system of equations arising from the linearization of a fully implicit scheme, at each time step. The main feature of this method is that the storage of the Jacobian matrix is completely eliminated, which leads to a low-storage algorithm. The viscous flux Jacobian matrices are replaced by their spectral radii. The convective flux are written with the Roe scheme instead of the Jameson scheme because of the dissipation term, the use of an inconsistent linearization having no consequence for steady computations. The Jacobian matrices which appear from the linearization of the centered fluxes are approximated with the numerical fluxes. The following system is obtained, for the iteration $n$ :

$$
\Delta w^{n}+\sum_{l=i, j, k}\left[\sigma_{l} \delta_{l} \mu_{l}\left(\Delta f_{l}^{n}\right)-\sigma_{l} \delta_{l}\left(\rho_{l}^{V} I_{d} \Delta w^{n}\right)-\sigma_{l} \delta_{l}\left(D_{l} \delta_{l} \Delta w^{n}\right)\right]=R_{e x p l}^{n}
$$

where $\Delta w^{n}=w^{n+1}-w^{n}, \sigma_{l}=\Delta t / \Delta x_{l}$, and $R_{\text {expl }}^{n}$ represents the explicit conservative residuals. For each direction $l, \rho_{l}^{V}$ is the viscous spectral radius, $\mu_{l}$ is the averaged operator, $\delta_{l}$ is the difference operator, $f_{l}$ is the physical convective flux and $D_{l}$ the dissipation matrix.

Then, the numerical dissipation matrices are replaced by their spectral radii and the system becomes matrix-free.

The implicit time-integration procedure leads to a system which can be solved directly or iteratively. The direct inversion can be memory intensive and computationally expensive. Therefore, an implicit relaxation procedure is preferred and the point Jacobi relaxation algorithm was chosen.

Concerning the turbulence transport equations, the diffusive flux Jacobian matrix is also replaced by its spectral radius. The source term needs a special treatment (Merci et al. 2000). Only the negative part of the source term Jacobian matrix is considered and replaced by its spectral radius. The system obtained is solved with a line-alternated Jacobi relaxation algorithm.

For steady state computations, convergence acceleration was obtained using a local time step and the full approximation storage (FAS) multigrid method proposed by Jameson (Jameson 1985). Forcing functions are defined on the coarser grids and added to the residuals used for the stepping scheme. The corrections computed on each coarse grid are transferred back to the finer one by trilinear interpolations. The turbulent equations are only solved on the fine grid and the computed eddy viscosity $\mu_{t}$ is transferred to the coarse grids. The multigrid algorithm is applied through a $V$-type cycle.

\subsection{Wall boundary condition}

At the wall, a no-slip condition was used coupled to a wall law treatment. It consists in imposing the diffusive flux densities, required for the integration process, in adjacent cells to a wall. The shear stress $\tau$ and the heat flux $q$ are obtained from the analytical velocity profile (4).

When using the wall law approach with the multigrid algorithm, the wall law boundary condition is applied on the fine grid and the classical no-slip condition is applied on the coarse grids. 


\subsection{Turbulence Models}

As the discretization scheme does not insure the positivity of the turbulent conservative variables, limiters are used to avoid negative $k$ or $\varepsilon$ values. These limiters are set equal to the corresponding imposed boundary values in the far field.

\section{COMPARISON TO EXPERIMENTS}

\subsection{Experimental conditions}

The DLR-F4 wing-body configuration has been studied experimentally in three different European wind tunnels (Redeker et al. 1987). The model is representative of a realistic transportaircraft configuration $(1,17 \mathrm{~m}$ span and $1.19 \mathrm{~m}$ body length) with a high aspect ratio transonic wing and an Airbus-type fuselage. The test case is defined as follows :

$M_{\infty}=0.75$, Mach number

$\alpha=0.93^{\circ}$, angle of attack

$T_{i}=300 K$, stagnation temperature

$R e_{c}=310^{6}$, Reynolds number based on the aerodynamic mean chord equal to $0.14 \mathrm{~m}$.

\subsection{Mesh}

The mesh used is a single block $C-O$ which defines half of the wing-body configuration. It contains 1026715 nodes, 257 nodes in the main direction, 85 in the normal direction of the wing and 47 in the spanwise direction along the wing. The outer boundaries are fixed at approximatively 4 fuselage-lengths (FL) upstream, one FL below and above the configuration and $3 \mathrm{FL}$ downstream. In the spanwise direction, the mesh covers one additional FL. A special contraction of the mesh is applied in the main flow direction, on the upper side of the wing, in the shock wave area.

The goal of the paper is not to obtain the best results with the finest mesh, but to obtain a reasonable quality of results at low cost. Thus, neither a grid refinement study nor a study of the influence of far-field distance were undertaken.

The $y^{+}$values, at the center of the first cells, vary between 1 and 30 on the wing and between 30 and 80 on the fuselage. Views of the mesh on the solid walls are given in Figure 1.

\subsection{Numerical parameters}

Computations are started from an uniform flow-field using a local time step. The numerical parameters used are :

- the CFL number, 10

- Jacobi iterations for the implicit stage, 14

- grids levels with multigrid algorithm, 2

- parameter $k_{2}$ of artificial dissipation: 0.5

- parameter $k_{4}$ of artificial dissipation: 0.032 for Jones-Launder and Wilcox models, 0.016 for other ones

- the farfield value of turbulent viscosity $\mu_{t_{\infty}}=0.1 \mu_{\infty}$

- the farfield value of turbulent kinetic energy $k_{\infty}=0.0012 \mathrm{~m}^{2} / \mathrm{s}^{2}$

- the farfield value of dissipation rate $\varepsilon_{\infty}=42 \mathrm{~m}^{2} / \mathrm{s}^{3}$

- the farfield value of specific dissipation $\omega_{\infty}=3500 \mathrm{~s}^{-1}$

For the computations, the flow is assumed to be fully turbulent. 


\subsection{Convergence and CPU time}

In Figure 2 is plotted the evolution of residuals for computations without multigrid algorithm and with two grids level. Both computations have been carried out with the $k-l$ model. The use of an implicit solver coupled with a multigrid strategy and wall functions allows to obtain a steady converged result with only 1000 iterations (about 55 minutes on a NEC-SX5 computer). For one-grid computations with two-equation models, the CPU time for 1000 iterations is about 2400 s, except the SST model, which needs about $6 \%$ more time. Two-grid computations need about $45 \%$ more computational time.

Faster computations could be obtained by using more efficient multigrid algorithm such as an adaptative-smoothing procedure (Drikakis et al. 2000).

\subsection{Aerodynamic coefficients}

The values of the aerodynamic coefficients of the complete configuration are reported in table 1 , for each turbulence model. The best lift coefficient is obtained with the Spalart-Allmaras model (only $1.5 \%$ of error). The Wilcox model over-predicts the lift coefficient (around 11\%) whereas the SST Menter model under-predicts it.

For all computations, the drag coefficient is largely under-estimated, especially with the Menter model (around $25 \%$ of error).

\subsection{Pressure coefficient}

The pressure coefficient distribution is shown in Figure 3, for three spanwise sections on the wing. The pressure level near the leading edge, on the suction side, is underestimated by all computations. At the closest section to the body, $y / b=0.185$, the best result is obtained with the $k-l$ model. The Spalart-Allmaras and Menter SST models predict a large and unrealistic separation at the trailing edge. This was also observed on DLR-F6 wing-body computations (Schwamborn 2006).

At the other sections, the shock location is well predicted with the Spalart-Allmaras model and a little downstream with the Smith model. As observed for two-dimensional computations, the Wilcox model predicts a shock location far away from the experimental data and the SST Menter model at an upstream location.

\subsection{Skin friction coefficient}

The evolution of the skin friction coefficient in the streamwise direction, on the suction side, is presented in Figure 4. There are no experimental values available, only an oil flow picture which does not show any shock-induced separation, which is well predicted by all computations. The SST Menter model gives the lowest skin friction levels. Near the wing root, the Spalart-Allmaras model is close to the Menter model.

\subsection{Deviation angle}

The evolution of the deviation angle $\beta_{0}$ (angle between the wall friction and the velocity at the boundary layer edge), on the suction side, is considered in Figure 5. There is a clearly pronounced peak at the shock location, the wall flow being deviated towards the wing tip. The SST model predicts large values of the deviation in the shock region and at the trailing edge. At the closest section to the body, as noticed on the pressure distribution, the Spalart-Allmaras and Menter models predict a large separation. 


\subsection{Vortical structures}

The skin friction lines over the half configuration are plotted in Figure 6, computed with the Smith model. A horseshoe vortex footprint around the wing root can be seen and also a vortical structure footprint at the trailing edge, near the root of the wing. The experimental oil flow picture also shows a vortex-like structure at the wing root but there is no flow visualization for the fuselage.

An enlargement of the wing/body junction, at the leading edge of the wing, is presented in Figure 7. We can well observe the horseshoe vortex around the root, the saddle point on the fuselage and the stagnation point on the wing. Such a separation has been evidenced by Furlano (Furlano et al. 2001) for 3D computations of an airfoil between walls, using the $k-l$ Smith model. In these results, the stagnation point is located on the fuselage. In the present case, it is certainly the sweep angle effect which moves the stagnation point on the wing. It can be noted that all turbulence models predict the same flow topology.

The horseshoe vortex feeds a vortical structure, called tornado vortex. The footprint of this vortex on the wing and the body is a focus, interpreted as a rolling up of the separation surface. This structure is generated by the interaction between the boundary layers of the wing and the fuselage (Delery 1992).

A detail of the wing/body junction, at the trailing edge of the wing, is presented in Figure 8, computed with the Smith model. The tornado vortex with the two foci are clearly evidenced. We note a third focus on the fuselage, interpreted as an unrolling up of the flow, which feeds the focus on the body, already fed by the horseshoe vortex. This third focus allows to preserve the volume of the stagnation flow of the vortical structure. Moreover, when using a very large close-up view of the trailing edge vicinity, it has been possible to identify a second saddle point and a node near the third focus, on the wing/body junction. Downstream of the tornado vortex, on the fuselage, is located a third saddle point which ends the horseshoe vortex surrounding the wing/body junction. Finally, we have three foci, three saddle points and two nodes (the stagnation point being equivalent to a node). To check the coherence of the three-dimensional separation, we use the following relation based on the critical-point theory and only valid for an isolated obstacle (Delery 1992, 2001) :

$$
\Sigma(\text { nodes and foci })-\Sigma(\text { saddle points })=2
$$

This relation is well respected but one should be careful, the identification of all critical points could be difficult.

When compared to the result obtained with the Smith model, the vortical structure predicted by the Wilcox model (Figure 9) and the Spalart-Allmaras model (Figure 10) presents a similar topology. This structure is a little less extended for the first model and largely overestimated by the second one. The big vortical structure with five foci predicted by the SST Menter model (Figure 11) is completely unphysical. This is certainly due to the SST correction based on the Bradshaw hypothesis, established only for two-dimensional turbulent boundary layers. This is consistent with conclusions derived from other studies on 3D configurations (Apsley and Leschziner 2001, Leschziner 2006).

\section{CONCLUSION}

An efficient compressible CFD method has been presented and applied to a realistic aircraft configuration. The combination of a low-storage implicit method, the FAS multigrid algorithm and wall functions allows to largely reduce the CPU cost. Presented numerical results verify the 
ability of the numerical tool to correctly predict the three-dimensional flow.

Four popular turbulence models have been tested and compared. Only indications of the models behavior can be provided considering the complexity of the flow and the experimental data available. When comparing the pressure coefficient distribution, the Smith and the SpalartAllmaras models provide the best results. Yet, near the wing root, the Spalart-Allmaras model predicts a large and unrealistic separation. As observed for two-dimensional computations over airfoils, the Wilcox model predicts a shock location far away from the experimental data and the SST Menter model at an upstream location.

With regard to the 3D separated flow generated on the wing-body junction, all models predict a coherent topological structure, except the SST Menter model which yields an unphysical vortical structure. This is certainly due to the rigid link the SST limiter establishes between the turbulence energy and shear stress. Even if global results are acceptable, the use of the SST correction for 3D flows should be clarified.

Additional works are in progress to simulate unsteady three-dimensional flows, to develop low dissipative numerical schemes and to pursue comparative analyses between turbulence models.

\section{References}

Apsley, D.D. and Leschziner, M.A., 2001. Investigation of advanced turbulence models for the flow in a generic wing-body junction. Flow, Turbulence and Combustion, 67, 25-55.

Chen, H.C. and Patel, V.C., 1988. Near-Wall Turbulence Models for Complex Flows Including Separation. AIAA Journal, 26(6), 641-648.

Craft, T.J., Gant, S.E., Iacovides, H. and Launder, B.E., 2004. A new wall function strategy for complex turbulent flows. Numerical Heat Transfer, part B, 45, 301-318.

Delery, J.M., 1992. Physics of vortical flows. Journal of Aircraft, 29(5), 856-876.

Delery, J.M., 2001. Robert Legendre and Henri Werle : toward the elucidation of threedimensional separation. Annual Review of Fluid Mechanics, 33, 129-154.

Drikakis, D., Iliev, O.P., and Vassileva, D.P., 2000. Acceleration of multigrid flow computations through dynamic adaptation of the smoothing procedure. Journal of Computational Physics, $165,566-591$.

Elsholtz, E., and John, D., 1997. 3D Navier-Stokes mesh generation and analysis of viscous flow solution around the DLR-F4 wing-body configuration. ECARP - Validation of CFD Codes and Assessment of Turbulence Models. Notes on Numerical Fluid Mechanics, 58, 69-80, Vieweg 1997.

Franke, M., Wallin, S., and Thiele, F., 2005. Assessment of explicit algebraic Reynolds-stress turbulence models in aerodynamic compuations. Aerospace Science and Technology, 9, 573581.

Furlano, F., Coustols, E., Plot, S., and Rouzaud, O., 2001. Steady and unsteady computations of flows close to airfoil buffeting: validation of turbulence models. Turbulence Shear Flow Phenomena, Stockholm, Sweden, June 27-29, 2001.

Goncalves, E., and Houdeville, R., 2001. Reassessment of the wall functions approach for RANS computations. Aerospace Science and Technology, 5, 1-14.

Jameson, A., Schmidt, W., and Turkel, E., 1981. Numerical simulation of the Euler equations by finite volume method using Runge-Kutta time stepping schemes. AIAA paper 81-1259, 14th Fluid and Plasma Dynamics Conference, Palo Alto, California, 1981.

Jameson, A.,1985. Multigrid Algoritms for Compressible Flow Calculations. Lecture Notes in Mathematics $\mathrm{n}^{\circ}$ 1228, Springer-Verlag, 1985.

Kalitzin, G., Medic, G., Iaccarino, G., and Durbin, P., 2005. Near-wall behavior of RANS turbulence models and implications for wall functions. Journal of Computational Physics, 204, $265-291$.

Kroll, N., Rossow, C.C., Becker, K., and Thiele, F., 2001. The MEGAFLOW project. Aerospace Science and Technology, 4, 223-237. 
Le Balleur, J.C., Girodroux-Lavigne, P., and Neron, M., 1997. Viscous-Inviscid Interaction Methods in 2D/3D-Steady/Unsteady-Problems. ECARP - Validation of CFD Codes and Assessment of Turbulence Models. Notes on Numerical Fluid Mechanics, 58, 197-222, Vieweg 1997.

Leschziner, M.A., 2006. Modelling turbulence separated flow in the context of aerodynamic applications. Fluid Dynamics Research, 38, 174-210.

Luo, H., Baum, J.D., and Lohner, R., 1998. A fast, matrix-free implicit method for compressible flows on unstructured grids. Journal of Computational Physics,146, 664-690.

Luo, H., Baum, J.D., and Lohner, R., 2001. An accurate, fast, matrix-free implicit method for computing unsteady flows on unstructured grids. Computers \& Fluids, 30, 137-159.

Menter, F.R., 1993. Zonal two equation $k-\omega$ turbulence models for aerodynamic flows. AIAA 93-2906, 24 ${ }^{\text {th }}$ Fluid Dynamics Conference, Orlando, Florida, July 6-9 1993.

Menter, F.R., 1994. Two-equation eddy-viscosity turbulence models for engineering applications. AIAA Journal, 32(8), 1598-1605.

Merci, B., Steelant, J., Vierendeels, J., Riemslagh, K., and Dick, E., 2000. Computational treatment of source terms in two-equation turbulence models. AIA A Journal, 38, 2085-2093.

Mohammadi, B., and Pironneau, O., Unsteady separated turbulent flows computation with walllaws and $k-\varepsilon$ model, Computational Methods in Applied Mechanical Engineering., 148, pp.393-405, 1997.

Olcmen, M.S., and Simpson, R.L., 1992. Perspective : on the near wall similarity of threedimensional turbulent boundary layers. Journal of Fluids Engineering, 114, 487-495.

Rakowitz, M., Eisfeld, B. Schwamborn, D., and Sutcliffe, M., 2003. Structured and unstructured computations on the DLR-F4 wing-body configurations. Journal of Aircraft, 40(2), 256-264.

Redeker, G., Muller, R., Ashill, R., Elsenaar, P.R., Schmitt, V., 1987. Experiments on the DLRF4 wing-body configuration in several European windtunnels. AGARD-FDP-Symposium, Naples, Italy, Sept.28-Oct.1, 1987.

Roe, P.L., 1981. Approximate Riemann solvers, parameters vectors, and difference schemes. Journal of Computational Physics, 43, 357-372.

Schwamborn, D., 2006. DLR F6. In: Haase, Aupoix, Bunge and Schwamborn, FLOMANIA - A European Initiative on Flow Physics Modelling, Notes on Numerical Fluid Mechanics and Multidisciplinary Design, 94, Springer Verlag.

Smith, B.R., 1994. A Near Wall Model for the $k-l$ Two Equation Turbulence Model. AIAA 94-2386, 25 ${ }^{\text {sh }}$ Fluid Dynamics Conference, Colorado Springs, Colorado, June 20-23 1994.

Spalart, P.R., and Allmaras, S.R., 1992. A one-equation turbulence model for aerodynamic flows. AIAA 92-0439, 30 th Aerospace Sciences Meeting, Reno, Nevada, January 6-9 1992.

Tatsumi, S., Martinelli, L., and Jameson, A., 1995. Flux-Limited Schemes for the Compressible Navier-Stokes Equations. AIAA Journal, 33, 252-261.

Tourrette, L., 1996. Assessment of turbulence models for the transonic flow around the DLR-F4 wing/body configuration. AIAA 96-2034, $27^{\text {th }}$ Fluid Dynamics Conference, New Orleans, Louisiana, June 17-20 1996.

Tsai, C.Y., and Whitney, A.K., 1999. Numerical study of three-dimensional flow separation for a 6:1 ellipsoid. AIAA 99-0172, $37^{\text {th }}$ Aerospace Sciences Meeting \& Exhibit, Reno, Nevada, January 11-14, 1999.

van Driest, E.R., 1951. Turbulent Boundary Layer in Compressible Fluids. J. Aeronaut. Sci., 18, 145-160.

van Driest, E.R., 1957. On Turbulent Flow Near a Wall. J. Aeronaut. Sci., 23, 1007-1011.

Viegas, J.R., and Rubesin, M.W., 1983. Wall-Function Boundary Conditions in the Solution of The Navier-Stokes Equations for Complex Compressible Flows. AIAA 83-1694, $16^{\text {th }}$ Fluid and Plasma Dynamics Conference, Danver, Massachussetts, July 12-14 1983.

Viegas, J.R., Rubesin, M.W., and Horstman, C.C., 1985. On the Use of Wall Functions as Boundary Conditions for Two-Dimensionnal Separated Compressible Flows. AIAA 85-0180, $23^{\text {rd }}$ Aerospace Sciences Meeting, Reno, Nevada, January 14-17, 1985. 
Wilcox, D.C., 1988. Reassessment of the scale-determining equation for advanced turbulence models. AIAA Journal, 26(11), 1299-1310. 


\section{List of Figures}

Fig1: Views of the mesh

Fig2: Convergence history

Fig3: Pressure coefficient

Fig4: Skin friction coefficient

Fig5: Deviation angle

Fig6: Skin friction lines - Smith model

Fig7: Detail of the leading edge - Smith model

Fig8: Detail of the trailing edge - Smith model

Fig9: Detail of the trailing edge - Wilcox model

Fig10: Detail of the trailing edge - SA model

Fig11: Detail of the trailing edge - SST model 
Table 1. Lift and drag coefficients

\begin{tabular}{ccccc}
\hline & LIFT & error & DRAG & error \\
\hline experiment & 0.602 & - & 0.0352 & - \\
$k-l$ & 0.638 & $6 \%$ & 0.0303 & $14 \%$ \\
$k-\omega$ & 0.67 & $11 \%$ & 0.0306 & $13 \%$ \\
$k-\omega$ SST & 0.571 & $5 \%$ & 0.0261 & $25 \%$ \\
Spalart-Allmaras & 0.61 & $1.5 \%$ & 0.0283 & $19.5 \%$ \\
\hline
\end{tabular}




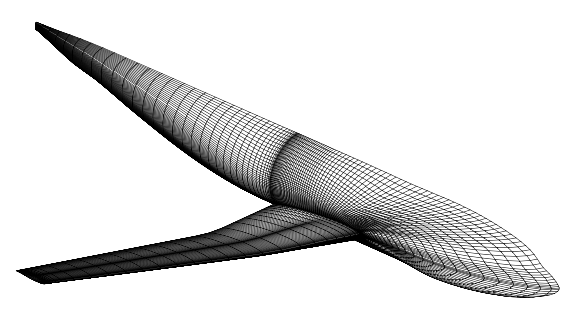

(a)

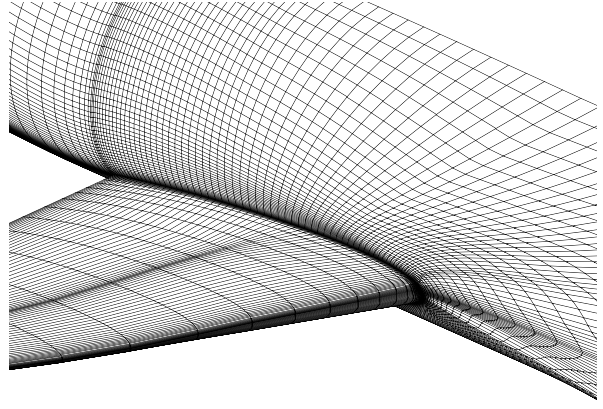

(b)

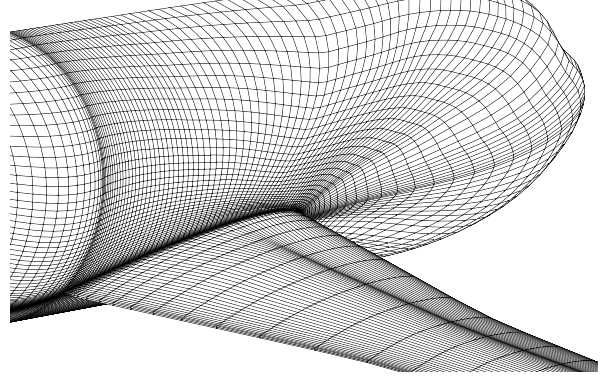

(c)

Figure 1. Views of the mesh 


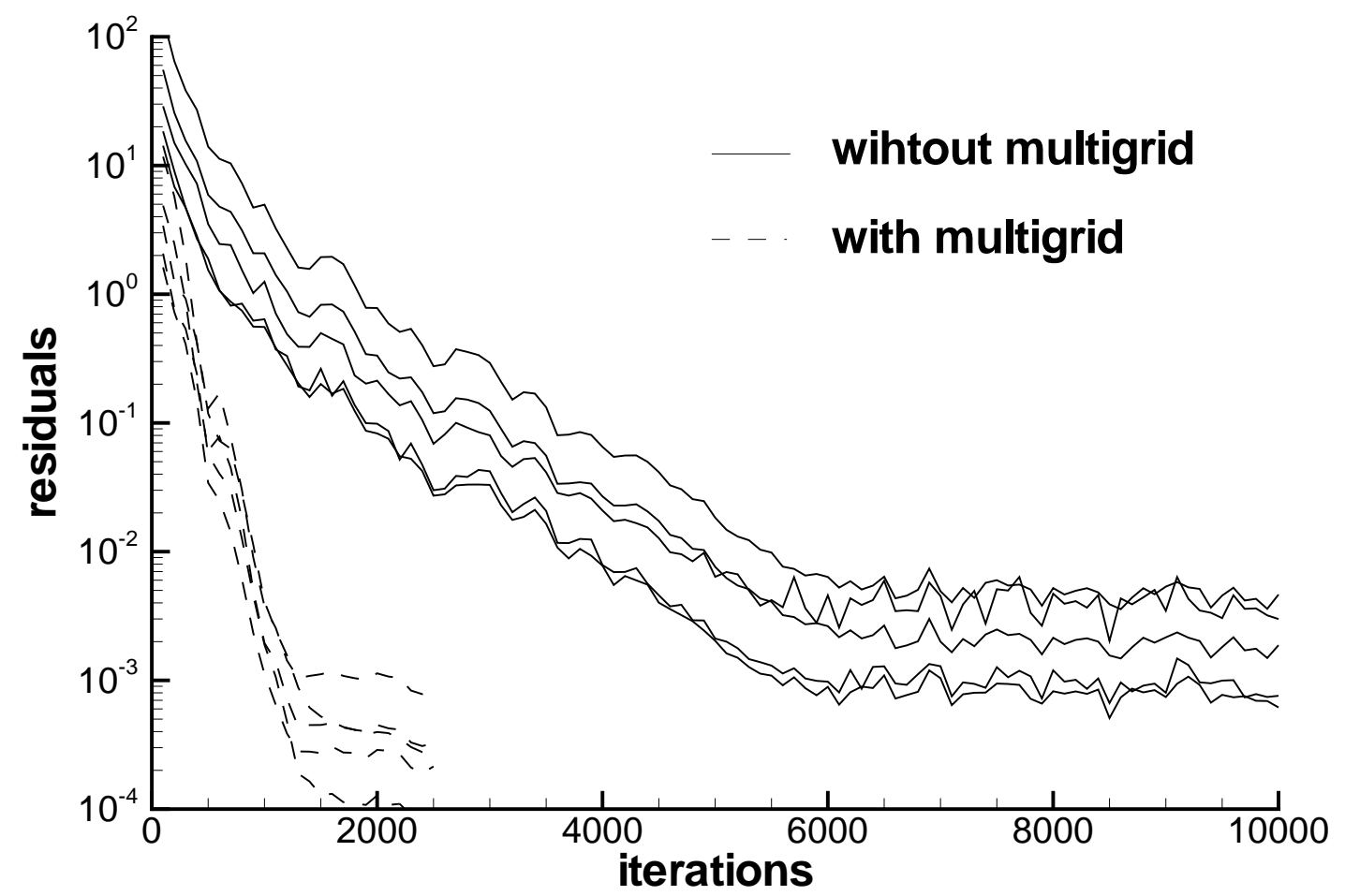

(a)

Figure 2. Convergence history 


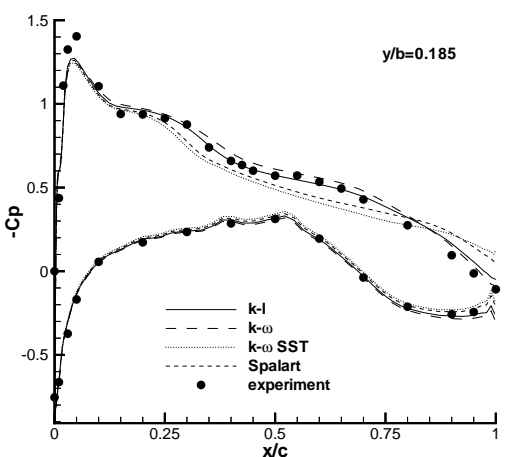

(a)

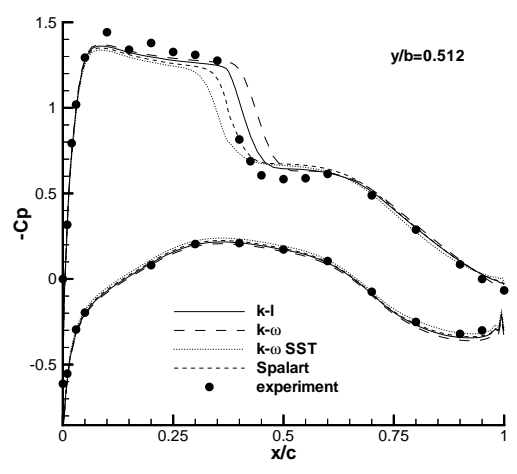

(b)

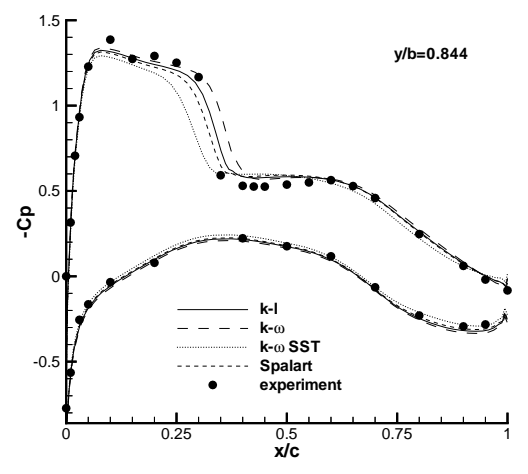

(c)

Figure 3. Pressure coefficient 


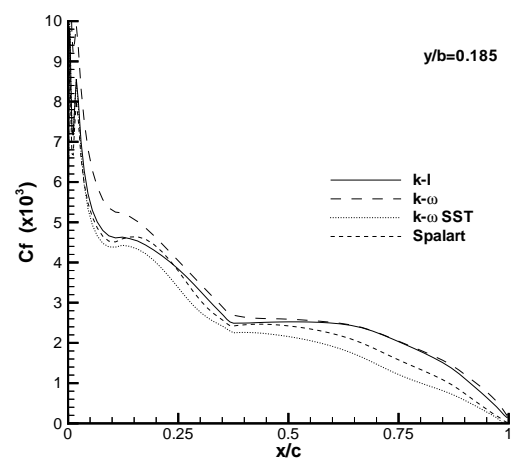

(a)

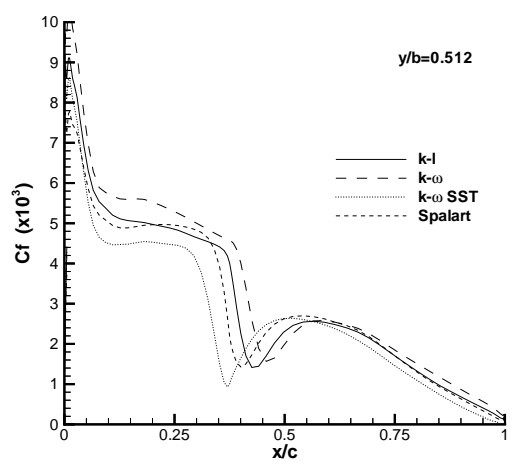

(b)

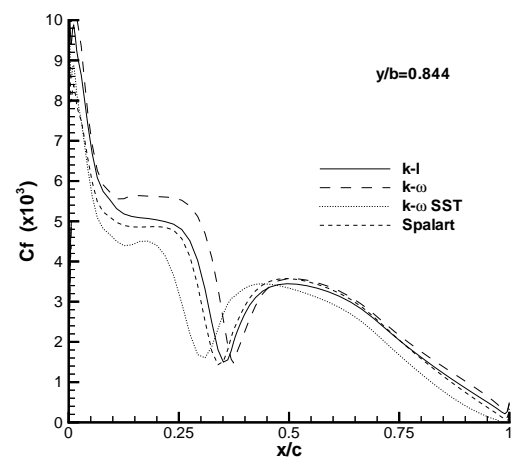

(c)

Figure 4. Skin friction coefficient 


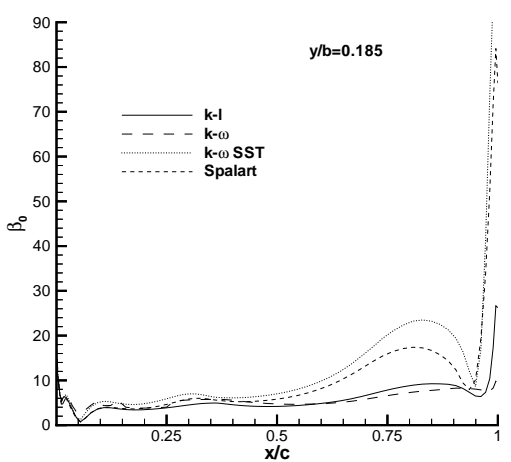

(a)

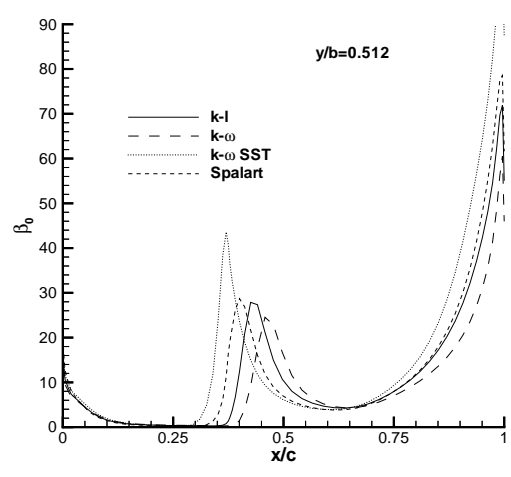

(b)

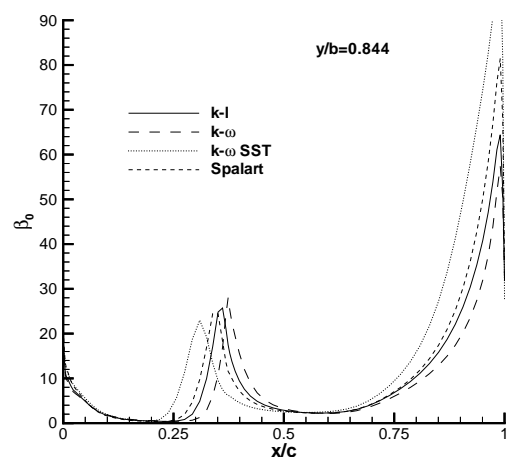

(c)

Figure 5. Deviation angle $\beta_{0}$ 


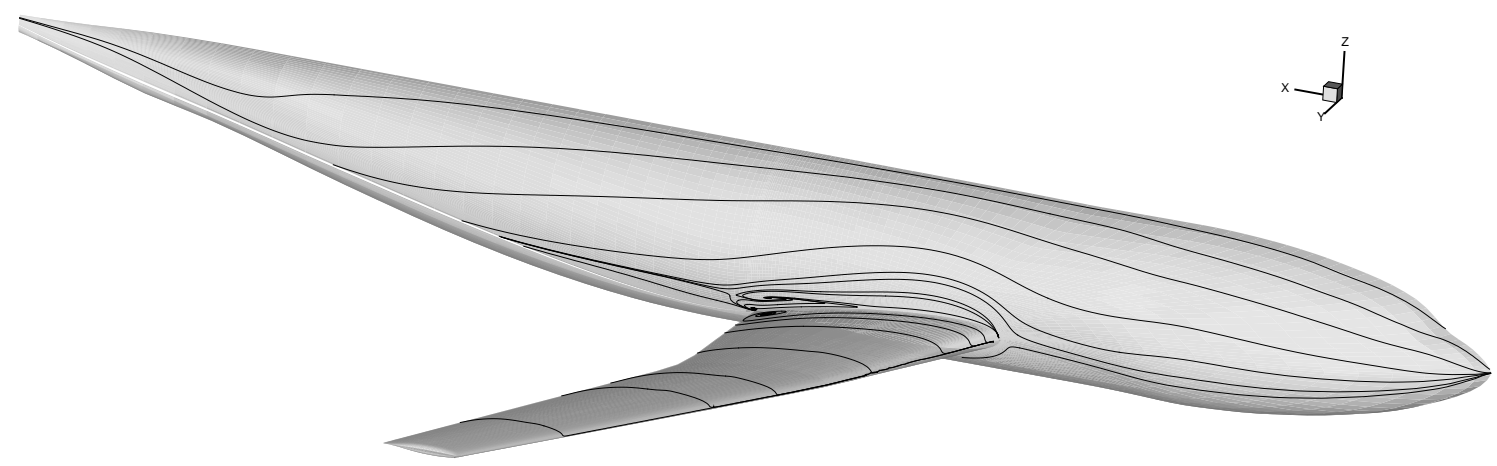

(a)

Figure 6. Skin friction lines - Smith model 


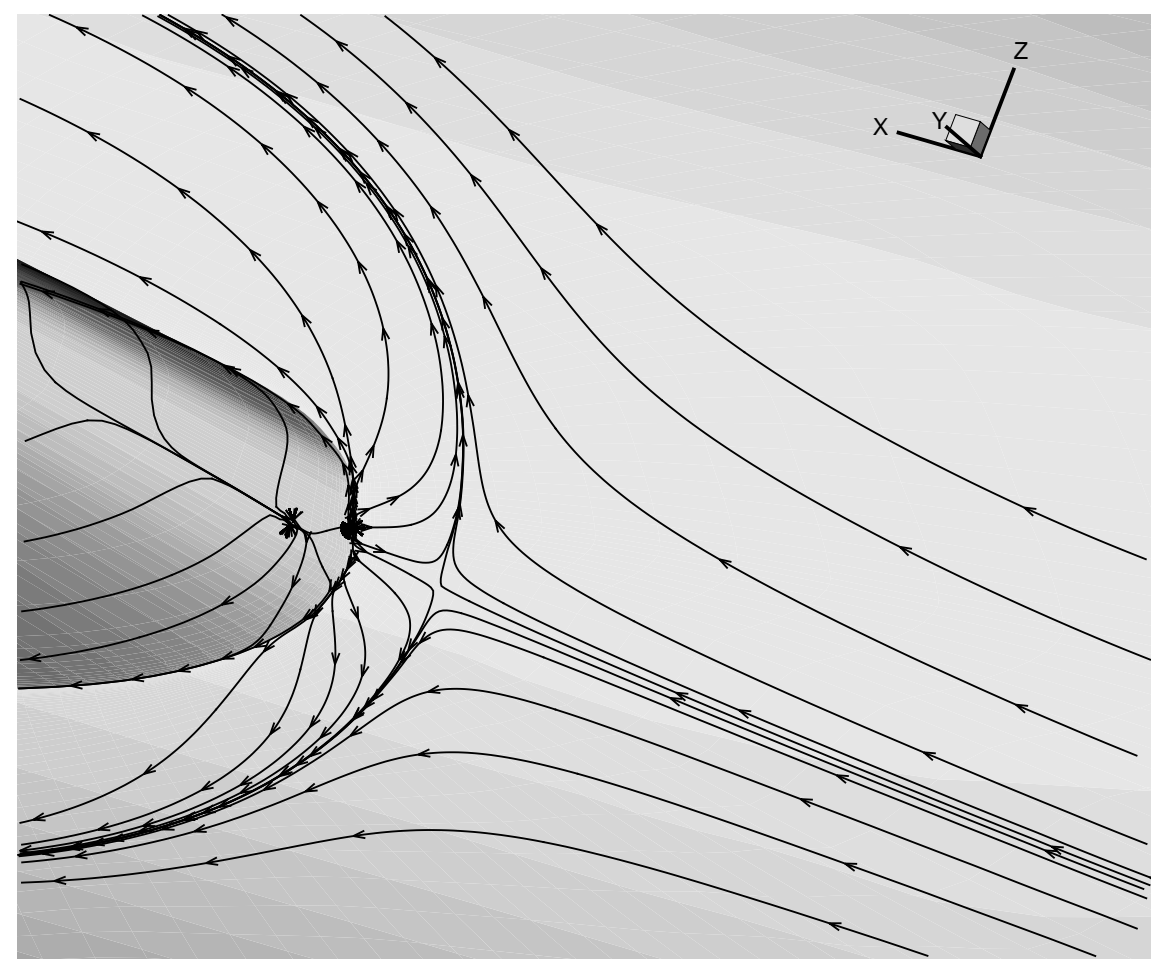

(a)

Figure 7. Detail of the leading edge - Smith model 


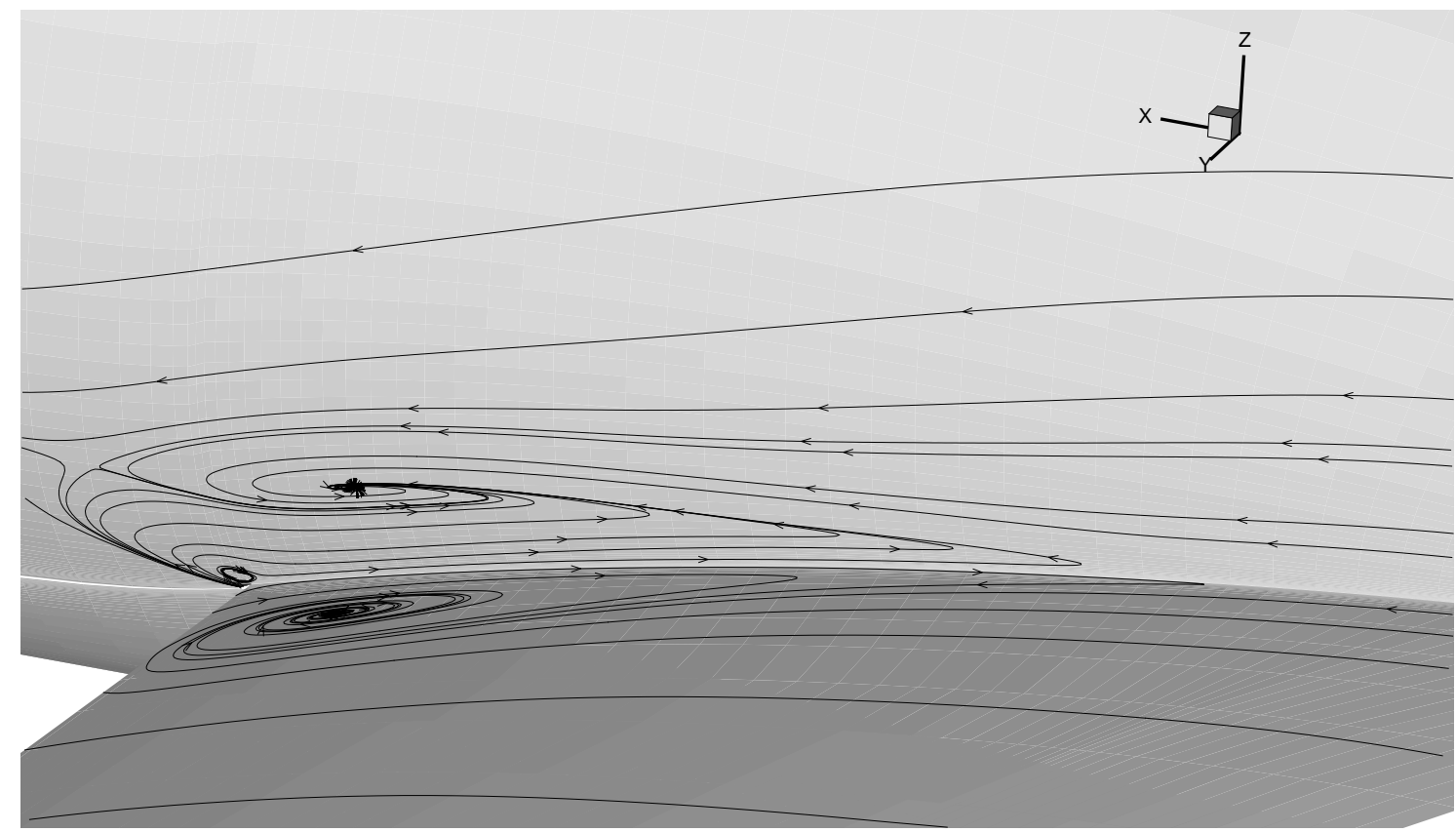

(a)

Figure 8. Detail of the trailing edge - Smith model 


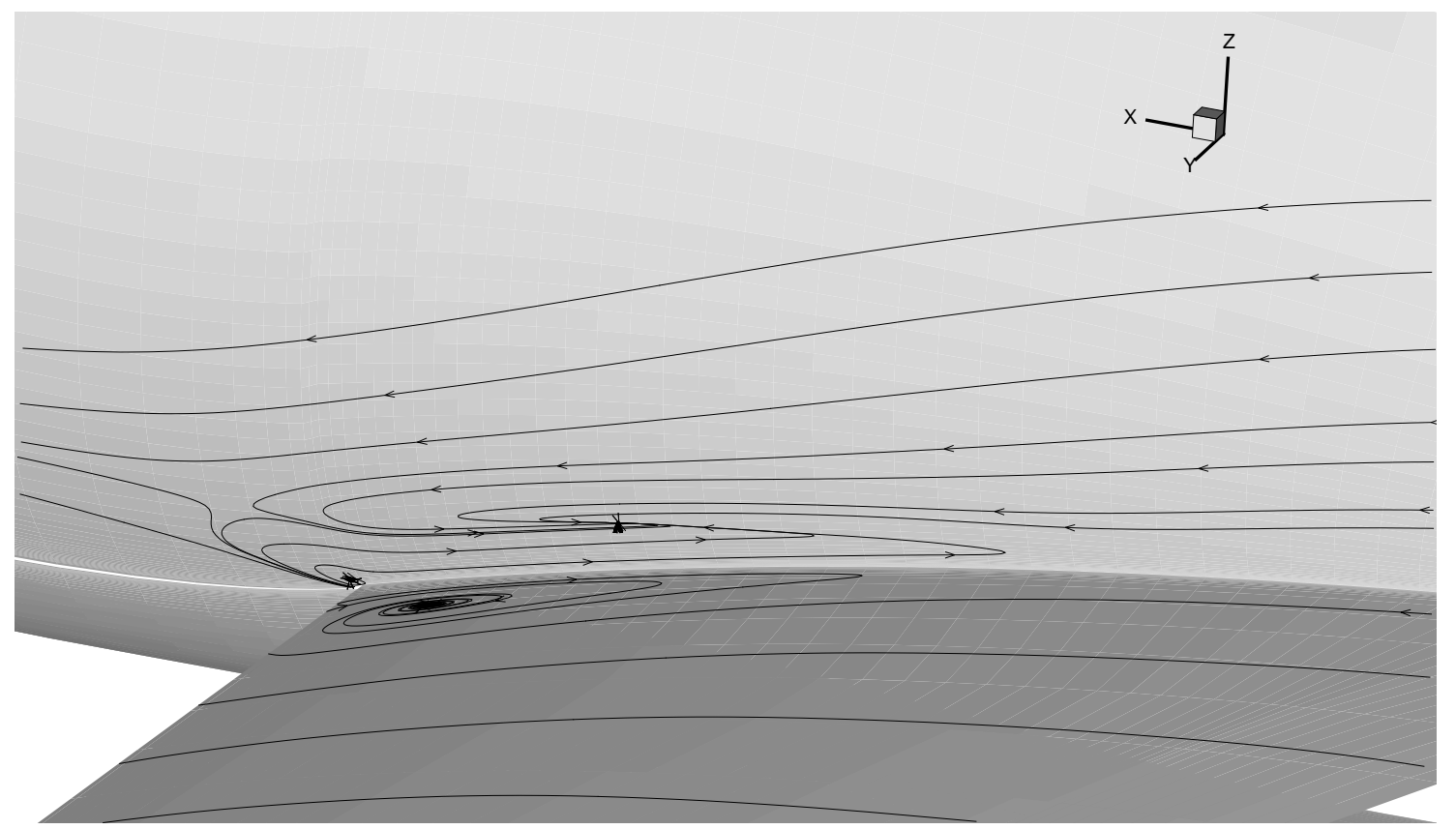

(a)

Figure 9. Detail of the trailing edge - Wilcox model 


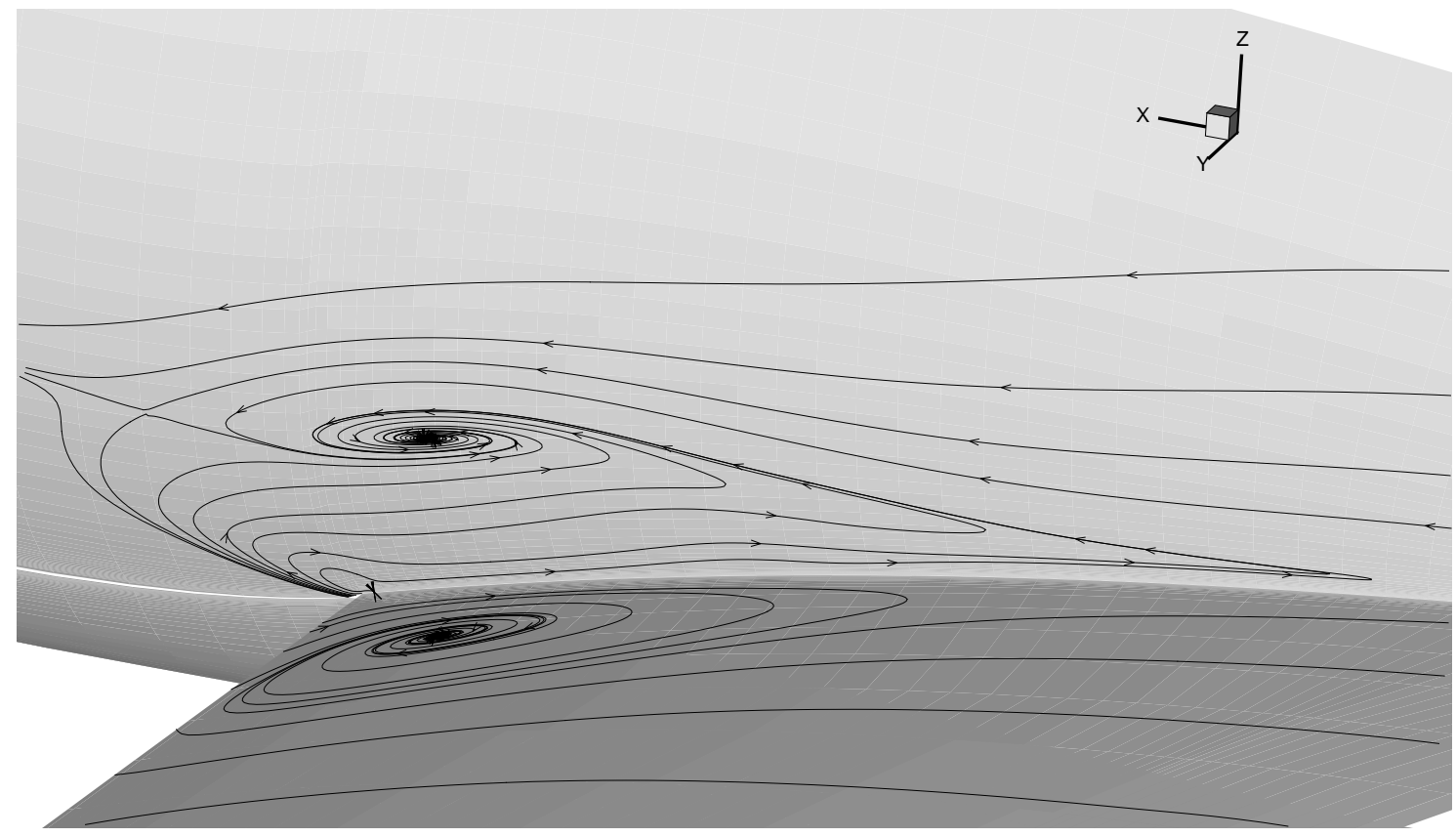

(a)

Figure 10. Detail of the trailing edge - Spalart-Allmaras model 


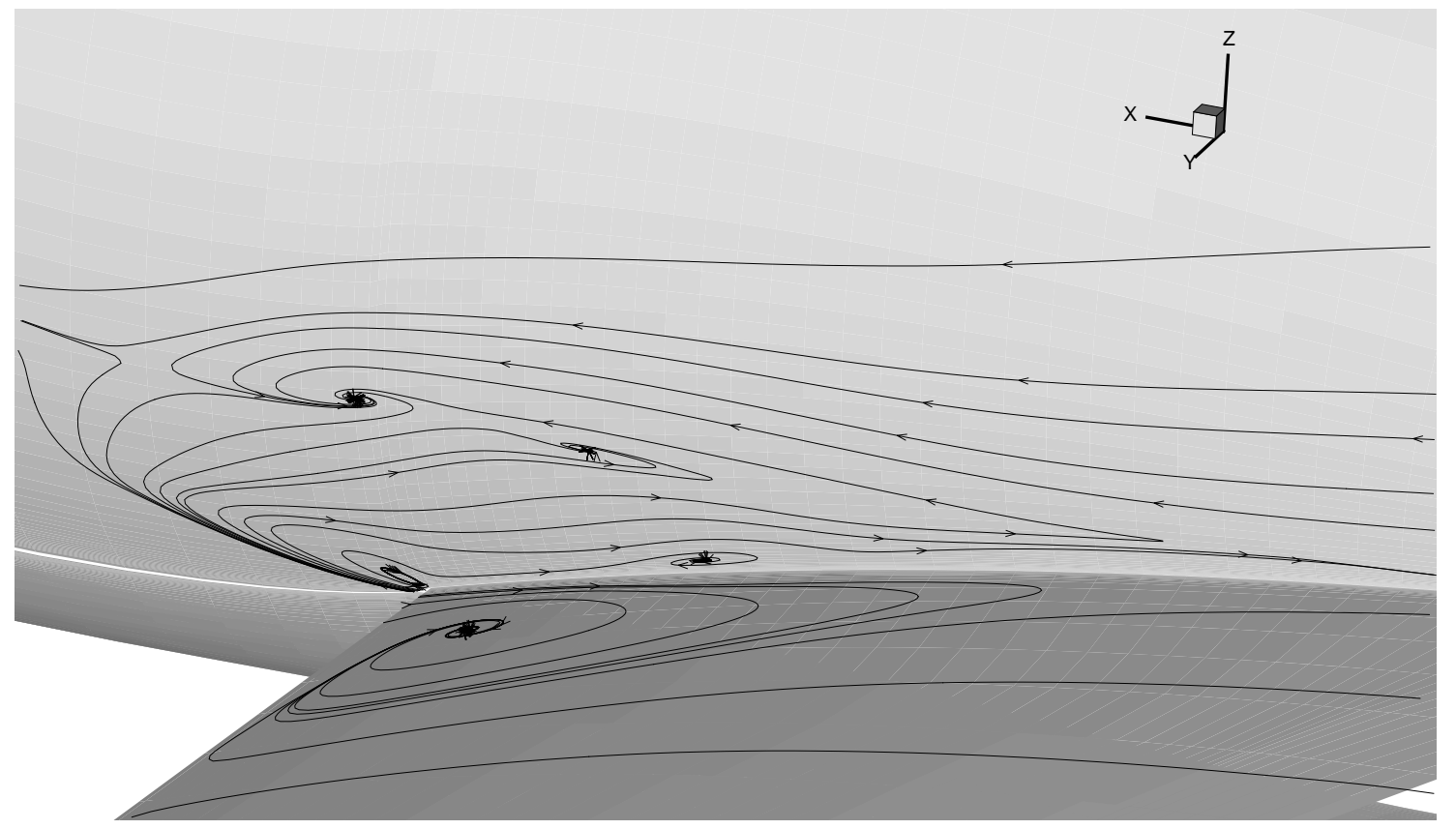

(a)

Figure 11. Detail of the trailing edge - Menter SST model 